\title{
Nearly strain-free heteroepitaxial system for fundamental studies of pulsed laser deposition: $\mathrm{EuTiO}_{3}$ on $\mathrm{SrTiO}_{3}$
}

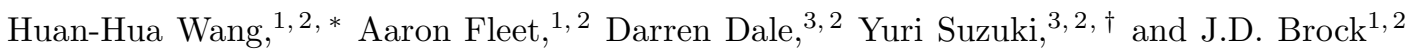 \\ ${ }^{1}$ School of Applied and Engineering Physics, Cornell University, Ithaca, NY 14853 \\ ${ }^{2}$ Cornell Center for Materials Research, Cornell University, Ithaca, NY 14853 \\ ${ }^{3}$ Department of Materials Science and Engineering, Cornell University, Ithaca, NY 14853
}

(Dated: May 9, 2019)

\begin{abstract}
High quality epitaxial thin-films of $\mathrm{EuTiO}_{3}$ have been grown on the (001) surface of $\mathrm{SrTiO}_{3}$ using pulsed-laser deposition. In situ x-ray reflectivity measurements reveal that the growth is twodimensional and enable real-time monitoring of the film thickness and roughness during growth. The film thickness, surface mosaic, surface roughness, and strain were characterized in detail using ex situ x-ray diffraction. The thickness and composition were confirmed with Rutherford BackScattering. The $\mathrm{EuTiO}_{3}$ thin-films grow two-dimensionally, epitaxially, pseudomorphically, with no measurable in-plane lattice mismatch.
\end{abstract}

\section{Introduction}

The growth of thin-films pervades modern electronic device fabrication. Indeed, the physical properties of thin-films are greatly influenced by their quality. And, the quality of a film is largely determined during its growth. Hence, optimizing growth processes is a significant theme of current research. Recently, thin-films of the transition metal oxides have been studied intensively due to their ferroelectric, ferromagnetic, and superconducting properties [1]. Pulsed-laser deposition (PLD) is widely used to deposit thin-films of these and other materials with non-trivial stoichiometries 2, 3, 4]. In PLD an intense pulse of laser light creates a "plume" of material by ablating it from a target. This plume exists for a few microseconds and consists of both ionic and neutral species. The energetic character of the plume distinguishes PLD from equilibrium growth techniques such as molecular-beam epitaxy (MBE) and chemical vapor deposition (CVD), while the enormous instantaneous flux distinguishes PLD from other energetic growth techniques such as sputter deposition or ion-beam assisted deposition (IBAD). While it is well-known that PLD can produce high quality epitaxial thin-films, our fundamental (atomic level) understanding of the PLD growth process remains incomplete.

Our long-term interest is identifying and studying the atomic scale, possibly energetic (i.e., non-thermal), mechanisms operant in PLD growth. Many factors, including differences in the symmetry of the substrate and thin-film lattices, a mismatch of the lattice constants, the partial pressure of oxygen, and the substrate temperature are known to strongly influence the growth process. Therefore, to isolate novel growth mechanisms, we require a nearly ideal thin-film/substrate combination that

\footnotetext{
* Current Address: Department of Physics and Astronomy, University of Georgia, Athens, GA 30602-2451

${ }^{\dagger}$ Current Address: Department of Materials Science and Engineering, University of California at Berkeley, Berkeley, CA 94720-1760
}

has consistent symmetry and that eliminates lattice mismatch. The objective of this study is to develop such a material system.

We have found that PLD of $\mathrm{EuTiO}_{3}$ (ETO) thin-films on $\mathrm{SrTiO}_{3}$ (STO) substrates satisfies these requirements. ETO and STO both have the cubic perovskite crystal structure at room temperature and both have the lattice constant $3.905 \AA[5,[6$, , 7]. The crystal structure of ETO is truly centrosymmetric, while STO exhibits only a tiny distortion from centrosymmetry [8]. In addition, neither material goes through a structural phase transition between $-220^{\circ} \mathrm{C}$ and typical PLD growth temperatures of $600-800^{\circ} \mathrm{C}[9]$. Previous X-ray studies in the literature indicate that oxygen-deficient solid solutions of $\mathrm{EuTiO}_{x}$ $(2.5<x<3)$ maintain the perovskite structure for the entire range of $x$ and do not report any change in the lattice constant [10, 11].

\section{Experimental Details}

These ETO/STO PLD studies are the first thinfilm growth experiments performed in the new thin-film growth/time-resolved x-ray diffraction facility in the G3 experimental station at the Cornell High Energy Synchrotron Source (CHESS), which utilizes x-rays generated by the Cornell Electron Storage Ring (CESR). The G3 experimental station combines a state-of-the-art ultra-high-flux synchrotron x-ray scattering facility with a fully featured PLD growth system, with the goal of performing time-resolved structural measurements of thinfilm growth. In these experiments, the (001) STO substrate sits at the origin of an x-ray diffractometer whose motions are integral components of the PLD growth chamber.

The ETO PLD target was synthesized by a solid-state reaction. Commercial $\mathrm{Eu}_{2} \mathrm{O}_{3}$ and $\mathrm{TiO}_{2}$ powders were mixed and compressed into a pellet. The pellet was then sintered at $1000^{\circ} \mathrm{C}$ for two days to obtain $\mathrm{Eu}_{2} \mathrm{Ti}_{2} \mathrm{O}_{7}$. The pellet was then reground into a powder and reduced in hydrogen gas at $1180^{\circ} \mathrm{C}$ into $\mathrm{EuTiO}_{3}$. The powder was

Typeset by REVTEX 
characterized using standard X-ray diffraction (XRD) techniques. The powder needed to be reground and reduced several times in order to obtain pure $\mathrm{EuTiO}_{3}$. The final powder diffraction profile, which does not exhibit any indication of a minority phase, is shown in Figure [1. The measured lattice constant is $3.896 \AA$, quite close to the previously reported value 12]. Finally, the purified powder was pressed into a pellet and vacuum sintered.

The substrates used in this study were commercially cut and polished (001) single-crystal wafers of $\mathrm{SrTiO}_{3}$. Each substrate was fixed to the stainless steel heater with silver paste as received from the supplier with no additional surface preparation. The target-substrate distance was $7 \mathrm{~cm}$. The heater temperature was measured by a thermocouple inserted into the heater. The substrate temperature is roughly $100^{\circ} \mathrm{C}\left(\right.$ at $650^{\circ} \mathrm{C}$ ) lower than the interior of the heater. Depositions were performed at heater temperatures ranging from $50^{\circ} \mathrm{C}$ to $650^{\circ} \mathrm{C}$.

The plume is created by a pulsed excimer laser (Lambda Physik, $248 \mathrm{~nm}$ ) focused on the (rotating) target with a fluence of $2 \mathrm{~J} / \mathrm{cm}^{2}$ and a repetition rate that ranged from 0.1 to $10 \mathrm{~Hz}$, in a background of $7 \times 10^{-6}$ Torr of $\mathrm{O}_{2}$.

In situ x-ray measurements were performed in realtime to monitor the growth process. Specifically, the time dependence of the intensity of the x-rays scattered at the anti-Bragg condition (equivalent to the out-ofphase condition in RHEED or LEED) exhibits intensity oscillations as the film grows. These oscillations can be caused both by variations in the roughness of the growth surface during layer-by-layer growth (as in RHEED studies) and by interference between $\mathrm{x}$-rays reflected by the film surface and the film/substrate interface (Kiessig fringes) 13, 14. This latter effect is only present in heteroepitaxy and is not usually observable in RHEED studies; however, it is very powerful as a timeresolved probe of film thickness. The intensities of the $\mathrm{Eu} \mathrm{L}_{2,3}$ emission lines were also monitored, providing an independent in situ measurement of the thickness of the growing ETO film [15, 16, 17].

After deposition, the film was cooled down to room temperature under the same oxygen pressure. The asgrown films are optically transparent. The thickness, surface roughness and crystal quality were characterized $e x$ situ with a standard rotating anode $\mathrm{Cu} \mathrm{K}_{\alpha}$ x-ray source. Rutherford Back Scattering (RBS) was used to characterize the film thickness and composition.

\section{Experimental Results}

Figure 2 shows an example of the time-dependent $\mathrm{x}$ ray intensity oscillations observed during PLD growth of ETO on STO $(001)$ at $650^{\circ} \mathrm{C}$ with a laser repetition rate of $0.1 \mathrm{~Hz}$. The deposition was stopped after 24 oscillation cycles. (Gaps in the time series occur during CESR injections.) In these measurements, the abrupt changes in intensity associated with each laser pulse (for example, as reported by G. Eres, et al. [18]) are not visible for two reasons. First, our pulses of material are smaller. During this growth, roughly 40 laser pulses were required to add a unit cell thick layer of ETO to the film. Second, the temporary x-ray optics available were not able to deliver the full $x$-ray beam, limiting the signal rate. Nevertheless, the observed intensity oscillations are clear evidence of two-dimensional growth - either layer-by-layer or stepflow. Growth mode phase diagrams for similar materials such as STO and $\mathrm{LaTiO}_{3}$ on STO suggest that the substrate temperature was not high enough for step flow growth and that the system should be in the layer-bylayer regime [19, 20]. The intensity oscillations, however, are Kiessig (or thickness oscillations) rather than roughness oscillations. Each oscillation corresponds to the addition of two rather than one unit cell of thickness. In the present case, roughness oscillations are likely to be difficult to observe for two reasons. First, the illuminated area on the sample is relatively large $(4 \mathrm{~mm}$ by $10 \mathrm{~mm}$ ) and includes portions of the substrate not in the center of the plume of ablated material. Consequently the growth rate varies across the illuminated area, causing the scattering from different regions of the film to oscillate at different frequencies. Second, the substrates did not receive any special treatment (e.g., etching) to prepare large, atomically flat terraces.

Figure [3] shows the absolute x-ray (specular) reflectivity as a function of scattered wave vector for the same film shown in Figure 2. These data were obtained ex situ using a conventional rotating anode-based $\mathrm{Cu} \mathrm{K}_{\alpha} \mathrm{x}$ ray source. The Kiessig fringes are clearly evident. The circles are the data and correspond to the integrated intensity obtained from a rocking scan taken at each $q$ point and then normalized to the incident beam intensity to obtain the absolute $\mathrm{x}$-ray reflectivity 21. The solid line is the best fit to the Parratt theory using the freeware package Parratt32 14]. The best fit value for the film thickness is $183.9 \pm 9.5 \AA$ with a RMS roughness of $4.7 \pm 1.5 \AA$ (the error bar represents a doubling of $\chi^{2}$ ). The inset to Figure 3 shows Rutherford Back Scattering (RBS) data taken on the same sample using singly charged helium ions at $1.165 \mathrm{MeV}$. Again, the circles are the data and the solid line is the best fit, which yielded a best fit value for the film thickness of $176 \AA$. The arrows indicate features in the RBS lineshape associated with A: europium, B: strontium, C: titanium, and D: oxygen.

Figure [4 shows an x-ray scattering scan on the same sample in the $(00 \ell)$ direction in the vicinity of the (001) STO Bragg peak. The solid line is a simple model consisting of a resolution limited Bragg peak for the STO substrate and a finite-size line-shape for the ETO thinfilm. Here we have assumed that the thin-film is 47 layers $(183.5 \AA)$ thick and that the ETO reciprocal lattice constant is $0.996 a^{*}$, where $a^{*}$ is the STO reciprocal lattice constant. The finite-size model describes the data quite well and the lattice mismatch in the out-of-plane direction is clearly visible in the raw data.

To characterize the crystal quality of the ETO thin- 
film, we performed detailed two-dimensional maps of the scattered x-ray intensity in the $(h 0 \ell)$ plane near the $(10$ 1) Bragg peak of STO and in the $(h k 0.996)$ plane. The first data set is shown in Figure [5. The data is plotted as a contour map with logarithmically spaced contours. This data demonstrates that the in-plane lattice constant of the ETO film is locked to that of the STO substrate. The streak from (101) towards smaller values of $\ell$ indicates that, consistent with the data in Figure 4 taken near the (0 0 1) Bragg peak, the out-of-plane ETO reciprocal lattice constant is roughly $0.4 \%$ smaller than that of the STO substrate. The smearing of the data in the $(10 \overline{1})$ direction is due to the resolution function of the diffractometer. The two-dimensional data set shown in Figure [6] also shows lines of constant scattered intensity but this time in the $\mathrm{H}-\mathrm{K}$ plane at $\ell=0.996$. The symmetry and location of the four ETO $\langle 101\rangle$ Bragg peaks clearly demonstrate the psuedomorphic growth of the ETO film.

To explore the roles of substrate temperature and laser pulse repetition rate, a series of growths were performed as the temperature and laser repetition rate were systematically varied. Varying the repetition rate from 0.1 to $10 \mathrm{~Hz}$ with the substrate temperature fixed at $600^{\circ} \mathrm{C}$ did not significantly affect the growth mode. Similarly, varying the substrate temperature from $600^{\circ}$ to $50^{\circ} \mathrm{C}$ while growing at $0.2 \mathrm{~Hz}$ did not affect the growth oscillations.

\section{Discussion}

In contrast to many of the rare-earth titanates $\mathrm{RTiO}_{3}$ $(\mathrm{R}=\mathrm{La}, \mathrm{Ce}, \mathrm{Nd}, \mathrm{Sm}, \mathrm{Y})$, which exhibit trivalent $\mathrm{R}$ and $\mathrm{Ti}, \mathrm{EuTiO}_{3}$ exhibits a divalent $\mathrm{Eu}$ and consequently a tetravalent Ti. In this sense ETO is very similar to the familiar tetravalent perovskite titanates $A \mathrm{TiO}_{3}(A=\mathrm{Ba}$, $\mathrm{Sr}, \mathrm{Ca})$. Therefore, in marked contrast to the $\mathrm{LaTiO}_{x}$ on STO system where the oxygen partial pressure had to be precisely tuned to the extremely reducing conditions required to stabilize $\mathrm{Ti}^{3+}[20]$, the conditions required to deposit stoichiometric $\mathrm{EuTiO}_{3}$ thin-films are relatively forgiving. Combined with the observed structural insensitivity to oxygen deficiency [6, 10], ETO is a very promising system for future studies of the oxidation of perovskite titanate thin-films 22, 23].

The characterization measurements, both in situ and ex situ, of the ETO films were very successful. First, the Kiessig fringe analysis of the specular reflectivity and the finite-size analysis of the (001) Bragg peak give consistent results for the film thickness. Next, the x-ray (Kiessig) and RBS film thickness measurements agree within experimental uncertainties. Finally, the x-ray measurements clearly demonstrate the smoothness of the film.

The data clearly demonstrate that, in the thickness range studied here, ETO thin films grow epitaxially and pseudomorphically in registry with the substrate. The in-plane lattice constant is observed to be locked to that of the STO substrate, while the out-of-plane lattice con- stant exhibits a small but measurable strain of approximately $0.4 \%$. If one assumes that the distortion of the ETO unit cell is volume conserving, then the lattice mismatch between ETO and STO is roughly $0.2 \%$. However, the following caveat needs to be kept in mind. Based on the growth conditions employed and information available in the literature, our ETO films are expected be oxygen deficient. And, one naively expects that an oxygen deficient sample should have a slightly dilated real space lattice constant due to additional electrons on the Ti sites. While the published literature does not report such an effect, the very small $(\sim 0.2 \%)$ strain we observe is not excluded by their data. Since, we have not measured the oxygen concentration, we are not able to specify exactly the strain state of a stoichiometric ETO film.

The observed smoothness of the films and the Kiessig oscillations indicate that the growth mode is twodimensional. The published growth phase diagrams for similar materials and our temperature and repetition rate studies suggest that the particular growth conditions utilized here are at too low a temperature to be in the stepflow regime. Therefore, we suggest that these growth conditions put the system somewhere near the layer-bylayer growth regime. However, since we were not able to observe the x-ray roughness oscillations, we cannot unambiguously determine the growth mode.

\section{Summary}

In summary, high quality epitaxial thin-films of $\mathrm{EuTiO}_{3}$ have been grown on the (001) surface of $\mathrm{SrTiO}_{3}$ using PLD. In situ x-ray reflectivity measurements reveal that the growth is two-dimensional and enable realtime monitoring of the film thickness and roughness during growth. The film thickness, surface mosaic, surface roughness, and strain were characterized in detail using ex situ x-ray diffraction. The thickness and composition were confirmed with Rutherford Back-Scattering. The $\mathrm{EuTiO}_{3}$ thin-films grow two-dimensionally, epitaxially, pseudomorphically, with no measurable in-plane lattice mismatch. The measured out-of-plane lattice mismatch is approximately $0.4 \%$,

\section{Acknowledgments}

We gratefully acknowledge the assistance of Frank DiSalvo, Dong Park, and John Sinnott during the target synthesis. This work was supported by the Cornell Center for Materials Research (CCMR) which is supported by the National Science Foundation under award DMR 0079992. A portion of this this work is based upon research conducted at the Cornell High Energy Synchrotron Source (CHESS) which is supported by the National Science Foundation and the National Institutes of Health/National Institute of General Medical Sciences under award DMR 9713424. The construction of the G- 
line facility was partially supported by the National Sci-

ence Foundation under awards DMR 9970838 and DMR
0114094 .
[1] M. Imada, A. Fujimori, and Y. Tokura, Reviews of Modern Physics 70, 1039 (1998).

[2] D. B. Chrisey and G. K. Hubler, eds., Pulsed Laser Deposition of Thin Films (John Wiley \& Sons, Inc., New York, 1994).

[3] P. R. Willmott and J. R. Huber, Reviews of Modern Physics 72, 315 (2000).

[4] D. H. Lowndes, D. B. Geohegan, A. A. Puretzky, D. P. Norton, and C. M. Rouleau, Science 273, 898 (1996).

[5] V. H. Holzapfel and J. Sieler, Zeitschrift Fur Anorganische Und Allgemeine Chemie 343, 174 (1966).

[6] G. J. McCarthy, W. B. White, and R. Roy, Journal of Inorganic \& Nuclear Chemistry 31, 329 (1969).

[7] T. Katsufuji and H. Takagi, Physical Review B 64, Art. No. 054415 (2001).

[8] B. Ravel and E. A. Stern, Physica B 209, 316 (1995).

[9] F. S. Galasso, Structure, Properties and Preparation of Perovskite-Type Compounds, vol. 5 of International Series of Monographs in Solid State Physics (Pergamon Press, Oxford, 1969).

[10] C.-L. Chien, Debenede.S, and F. D. S. Barros, Physical Review B 10, 3913 (1974).

[11] G. J. McCarthy, W. B. White, and R. Roy, Materials Research Bulletin 4, 251 (1969).

[12] J. Brous, I. Fankuchen, and E. Banks, Acta Crystallographica 6, 67 (1953).
[13] H. Kiessig, Ann. Phys. 10, 769 (1931).

[14] J. Als-Nielsen and D. McMorrow, Elements of Modern $X$-ray Physics (Wiley, New York, 2001).

[15] R. L. Headrick, S. Kycia, Y. K. Park, A. R. Woll, and J. D. Brock, Physical Review B (Condensed Matter) 54, 14686 (1996).

[16] R. L. Headrick, S. Kycia, A. R. Woll, J. D. Brock, and M. V. R. Murty, Physical Review B (Condensed Matter) 58, 4818 (1998).

[17] A. R. Woll, R. L. Headrick, S. Kycia, and J. D. Brock, Physical Review Letters 83, 4349 (1999).

[18] G. Eres, J. Z. Tischler, M. Yoon, B. C. Larson, C. M. Rouleau, D. H. Lowndes, and P. Zschack, Applied Physics Letters 80, 3379 (2002).

[19] J. Song and Y. Jeong, Solid State Commuications 125, 563 (2003).

[20] A. Ohtomo, D. A. Muller, J. L. Grazul, and H. Y. Hwang, Applied Physics Letters 80, 3922 (2002).

[21] D. Gibbs, B. M. Ocko, D. M. Zehner, and S. G. J. Mochrie, Physical Review B 38, 7303 (1988).

[22] X. D. Zhu, W. D. Si, X. X. Xi, Q. Li, Q. D. Jiang, and M. G. Medici, Applied Physics Letters 74, 3540 (1999).

[23] X. D. Zhu, W. D. Si, X. X. Xi, and Q. D. Jiang, Applied Physics Letters 78, 460 (2001). 


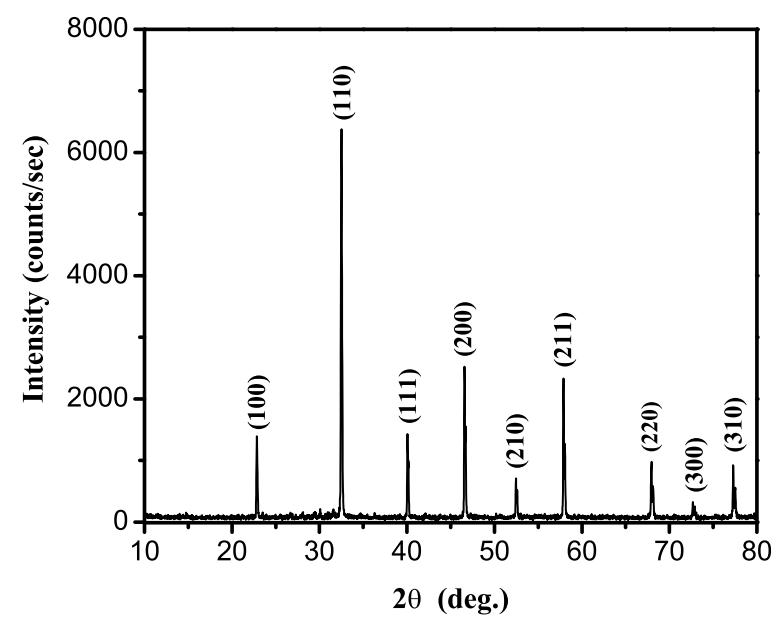

FIG. 1: XRD scan of $\mathrm{EuTiO}_{3}$ powder sample before being sintered into a pellet for use as a PLD target. All of the visible peaks have been indexed to the cubic perovskite structure. Note the absence of any peaks associated with a minority phase.

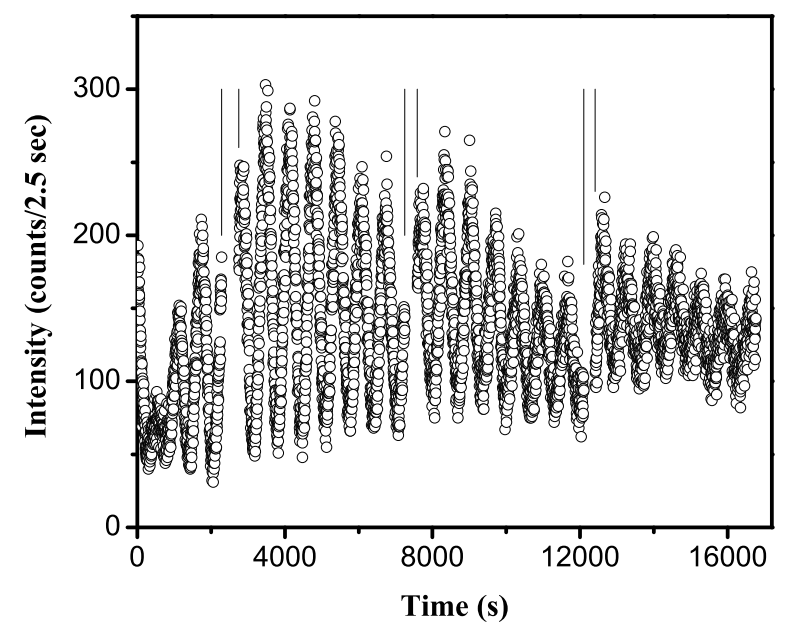

FIG. 2: Oscillations of the intensity of specularly reflected $\mathrm{x}$-rays at the anti-Bragg position during PLD growth of $\mathrm{EuTiO}_{3}$ on $\mathrm{SrTiO}_{3}$ (001). The oscillations are Kiessig fringes. Each oscillation corresponds roughly to adding two layers of $\mathrm{EuTiO}_{3}$. Gaps in the time series correspond to CESR injections. 


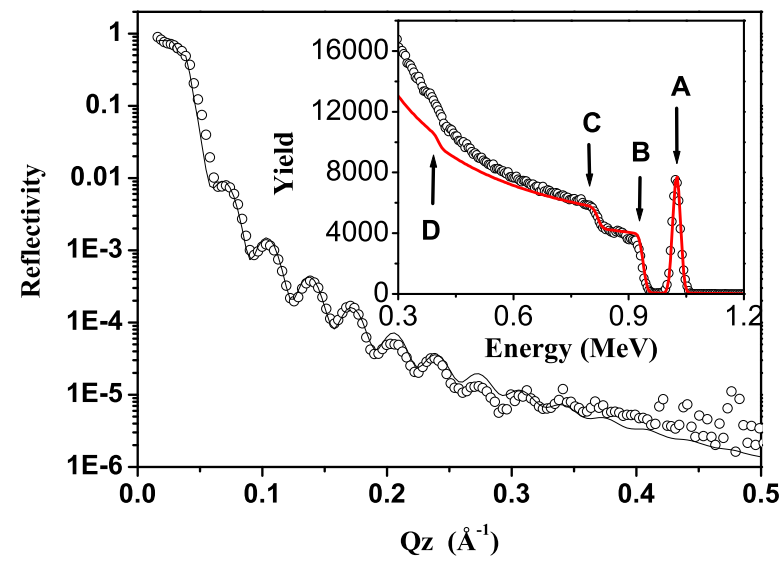

FIG. 3: Absolute specular reflectivity of $\mathrm{EuTiO}_{3}$ film on $\mathrm{SrTiO}_{3}$. Each circle represents the area under the specular peak in a transverse scan. The solid line is the best fit to a Parratt line-shape. Inset: RBS scan of the same film. Circles are the data and the solid line is the model calculation. The arrows indicate features in the RBS lineshape associated with A: europium, B: strontium, C: titanium, and D: oxygen.

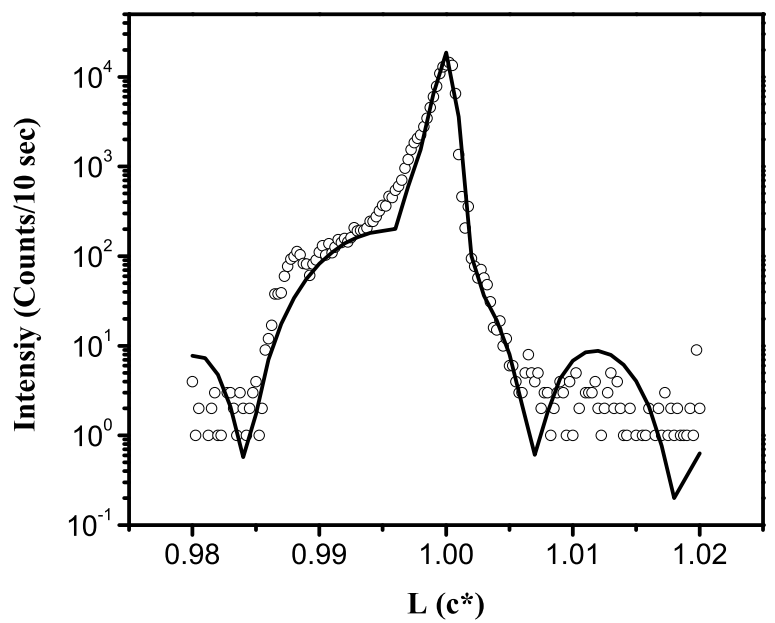

FIG. 4: Rod scan through (001) Bragg peak of $\mathrm{SrTiO}_{3}$ and finite-thickness broadened thin-film "Bragg" peak. Solid line is a simple model calculation consisting of a resolution limited STO Bragg peak and a simple finite-size line-shape for the ETO film. 


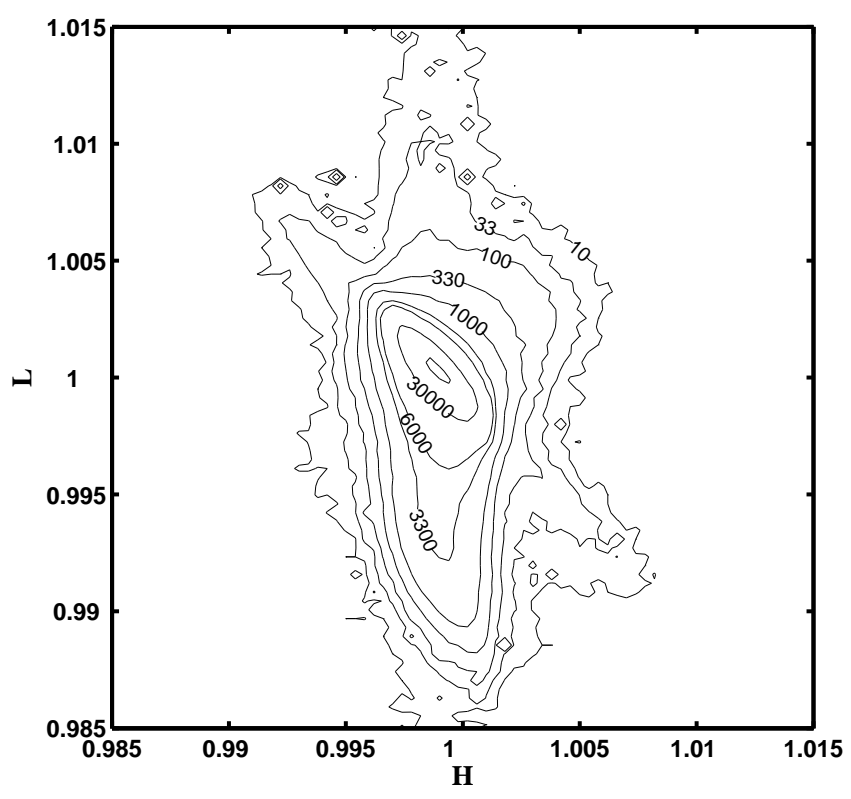

FIG. 5: Scattered intensity in the H-L plane near the (101) Bragg peak of $\mathrm{SrTiO}_{3}$. Lines are contours of constant scattered intensity. ETO Bragg peak is roughly at (1 0 0.996).

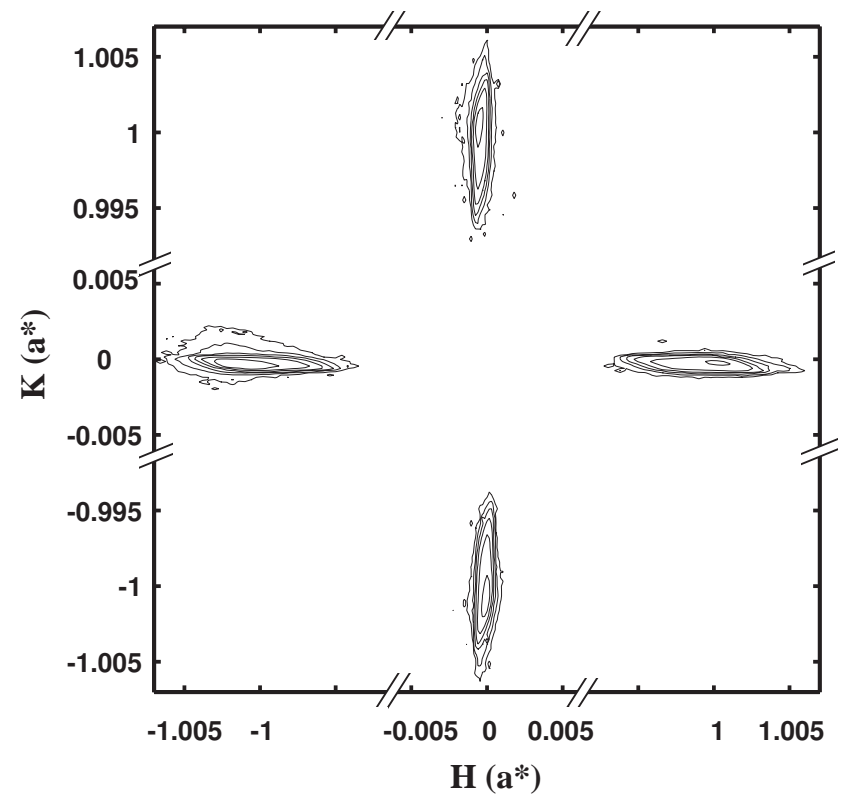

FIG. 6: Scattered intensity in the $\mathrm{H}-\mathrm{K}$ plane of $\mathrm{SrTiO}_{3}$ at $\ell=0.996$. Lines are contours of constant scattered intensity. 